\title{
Auswirkungen der Grundwasser- bewirtschaftung auf die Grund- und Oberflächenwasserqualität des 2. und 20. Wiener Gemeindebezirkes
}

\section{The impact of groundwater management on the quality of ground and surface water in the 2nd and 2oth districts of Vienna}

\author{
von S.SCHUSTER
}

KURZFASSUNG/SUMMARY

Das Grundwasser im 2. und 20. Bezirk wird nach Errichten einer Dichtwand alleinig durch 25 Bewirtschaftungsbrunnen entlang der Donau reguliert. Online Wasserqualitätsmessstellen, welche kontinuierlich Donau- und Uferfiltrat auf chemische Parameter untersuchen, dienen neben der in regelmäßigem Intervall stattfindenden Beprobung und Untersuchung von Grundwassermessstellen einer qualitativen Früherkennung von Verschlechterungen sowie generell der qualitativen Überprüfung und Beweissicherung von Grund- und Oberflächengewässern. Nach nunmehr 10 Jahren Grundwasserbewirtschaftung konnte durch den direkten Vergleich der Zeitperioden vor Stauerrichtung ,IST-Zustand“ und der Periode nach Vollstauerrichtung die chemischen Veränderungen der Grund- und Oberflächengewässer aufgezeigt werden. Im Nahbereich der Donau stellte sich im Grundwasser ein deutlicher Donau-beeinflusster Charakter ein, welcher sich langsam und abgeschwächt, jedoch messbar bis ins Hinterland auswirkt. Durch die strenge qualitative Kontrolle des zur Versickerung gebrachten Uferfiltrates werden Kontaminationen verhindert, sodass sich langfristig bestehende lokale Verunreinigungen verdünnen bzw. natürlich abbauen. Erstmalig auf der Welt wurde unter immensem finanziellem Aufwand ein qualitatives Messprogramm etabliert, welches der laufenden Qualitätskontrolle sowie der nachhaltigen Beweissicherung der Grund- und Oberflächen-

wasserqualität zweier Wiener Gemeindebezirke dient. Veränderungen der Wasserqualität und Auswirkungen dieses Systems sind in diesem Beitrag zusammengefasst.

Since the provision of the impervious diaphragm for the power development on the River Danube, the water table in the 2nd and 2oth districts of Vienna has been controlled only through 25 wells located along the river. On-line water quality monitoring stations, which continuously check

Danube and river-bank filtrates for chemical parameters, serve to ensure early identification of deterioration in quality, thus supplementing the regular sampling and analyses conducted at groundwater measuring stations. In addition, they serve for a general qualitative check and recording of evidence for ground and surface water. After 10 years' groundwater management, direct comparison between the conditions prior to and after impoundment shows the chemical changes that have taken place in the ground and surface water. In the vicinity of the Danube, the ground water has come to reflect a distinct influence from the river water, and this gradually decreases towards the hinterland. Strict qualitative control of the river-bank filtrate which seeps into the ground prevents contamination, so that existing local contaminations can in the long term become diluted or degraded by natural processes. An extremely expensive qualitative monitoring programme has been established as a worldwide first. This provides regular quality control and recording of evidence for the quality of ground and surface water in two districts of Vienna. Changes in water quality and the effects of this system are summarised in this report.

\section{EINLEITUNG}

Die Grundwasserbewirtschaftung des 2. und 20. Bezirkes umfasst 25 Brunnenanlagen zur direkten Entnahme von Donauuferfiltrat, 21 Versickerungsbrunnen zur Dotation des Grundwassers hinter den errichteten Dichtwänden und 21 Messstellen entlang der Häuserfront am Handelskai (Hinterland) (DREHER und GUNATILAKA, 1997). Weiters werden im Zuge der qualitativen Beweissicherung der Grundwasserbewirtschaftung laut Umfang der qualitativen hydrologischen Beweissicherung für das Kraftwerk Wien Freudenau auch hygienisch relevante Messstellen (HRM) im Hinterland des 2. und 20. Bezirkes analysiert. Online-Wasserqualitätsmessstationen von Donau und Uferfiltrat dienen der Frühwarnung allfälliger Wasserverunreinigungen und vervollständigen die Gesamtbeweissicherung für dieses Teilgebiet (GUNATILAKA und DREHER, 1997).

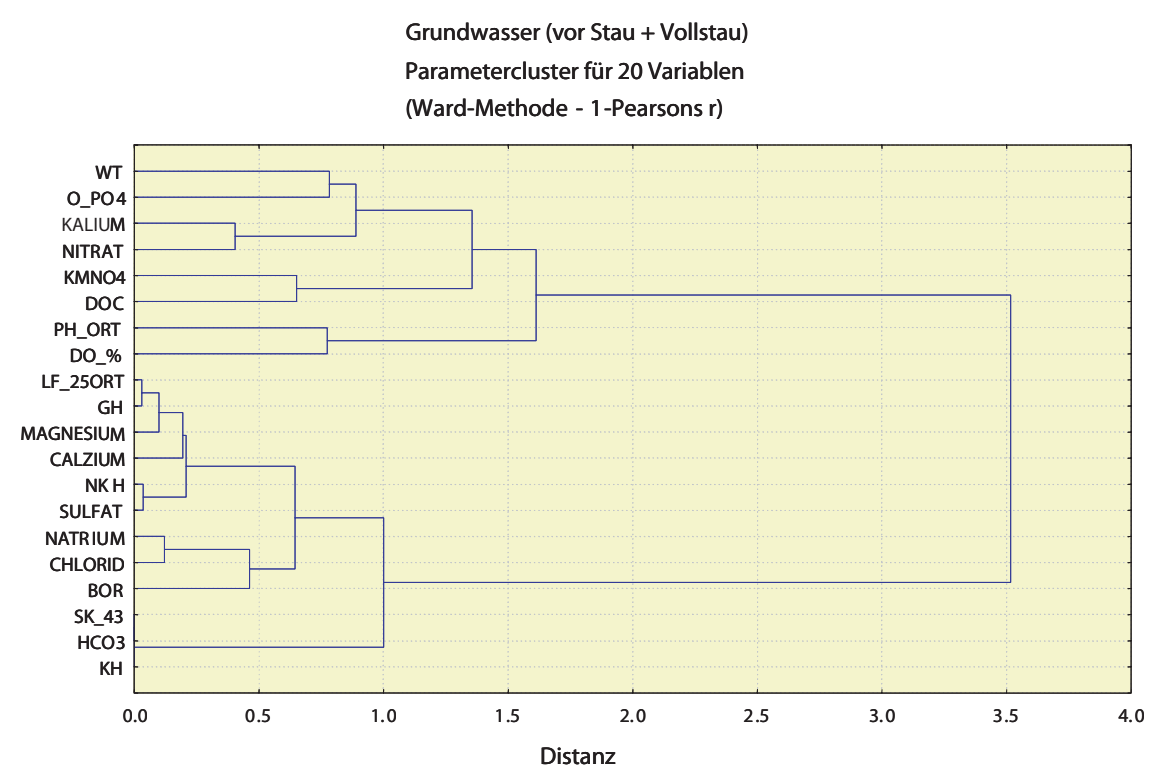

Abb. 1. Gruppenbildung von 20 vordefinierten Parametern über alle Grundwasserprobenahmestellen gepoolt mittels Clusteranalyse. Ähnlichkeiten drücken sich durch kürzere Distanzen der Seitenäste aus. 


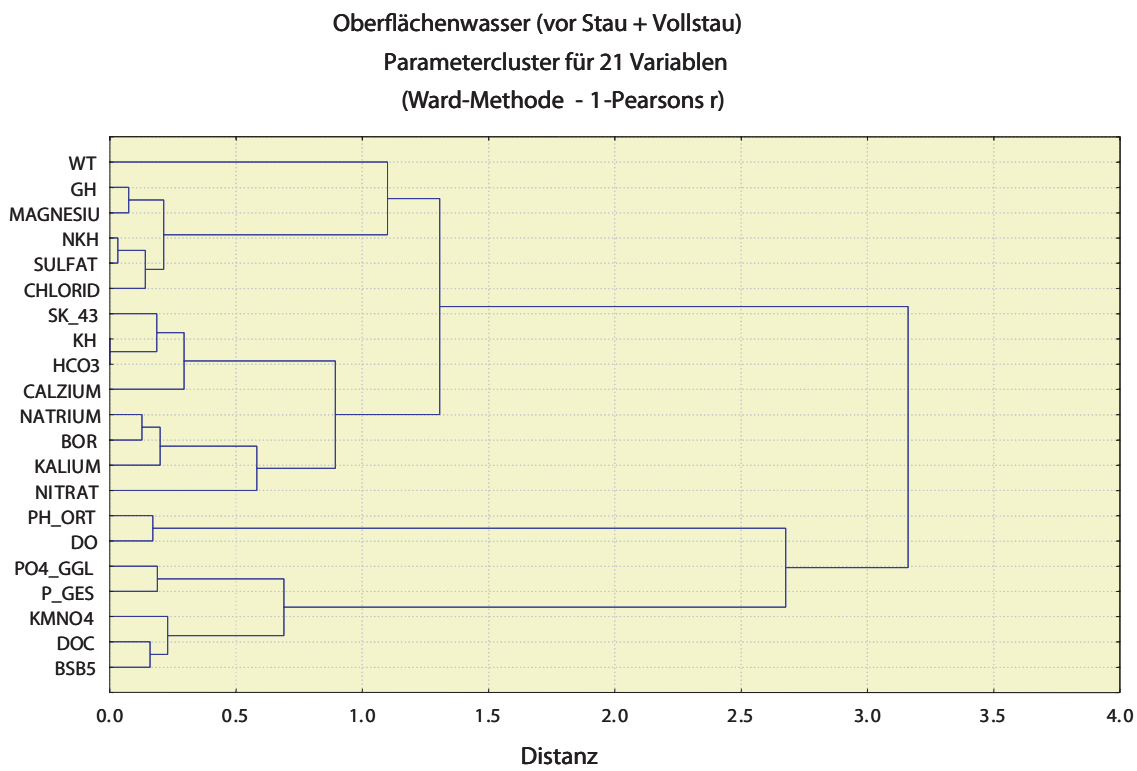

Abb. 2. Gruppenbildung von 21 vordefinierten Parametern über alle Oberflächenwasserprobenahmestellen gepoolt mittels Clusteranalyse. Oberflächenwasser relevante Parameter wie BSB5 und DOC sind deutlich mit der Oxidierbarkeit (hier als Permanganatverbrauch dargestellt) korreliert. Diese Gruppe für sich zeigt wiederum eine große Ähnlichkeit mit den Phosphatfraktionen. Deutlich davon abgetrennt ist die Gruppe der Hauptkationen und -anionen.
Ziel des qualitativen Beweissicherungsprogramms ist das rechtzeitige Erkennen einer Grundwasserverunreinigung im 2. und 20. Wiener Gemeindebezirk sowie das Abstellen des Dotationsbetriebes bei Verschlechterung der Qualität des Donauwassers oder des Uferfiltrates vor oder nach der Inbetriebnahme der Grundwasserbewirtschaftung der 25 an der Donau situierten Brunnenanlagen. Überschreitet ein Qualitätsparameter einen definierten und vorgeschriebenen Grenzwert (Abbruchkriterium), ist die Dotation einzustellen und die in der Betriebsordnung vorgesehenen Maßnahmen sind anzuwenden.

Nur wenn die Konzentrationen der untersuchten Parameter der Proben aus den Entnahmebrunnen unterhalb der Abbruchkriterien (in der Folge AK genannt) bleiben, darf Uferfiltrat aus der Donau im Hinterland über die Schluckbrunnen zur Versickerung gebracht werden.

Die Messstellen werden aufgrund ihrer geografischen Lage und ihrer Wasserqualität zu zwei Großgruppen mit Untergruppen zusammengefasst:
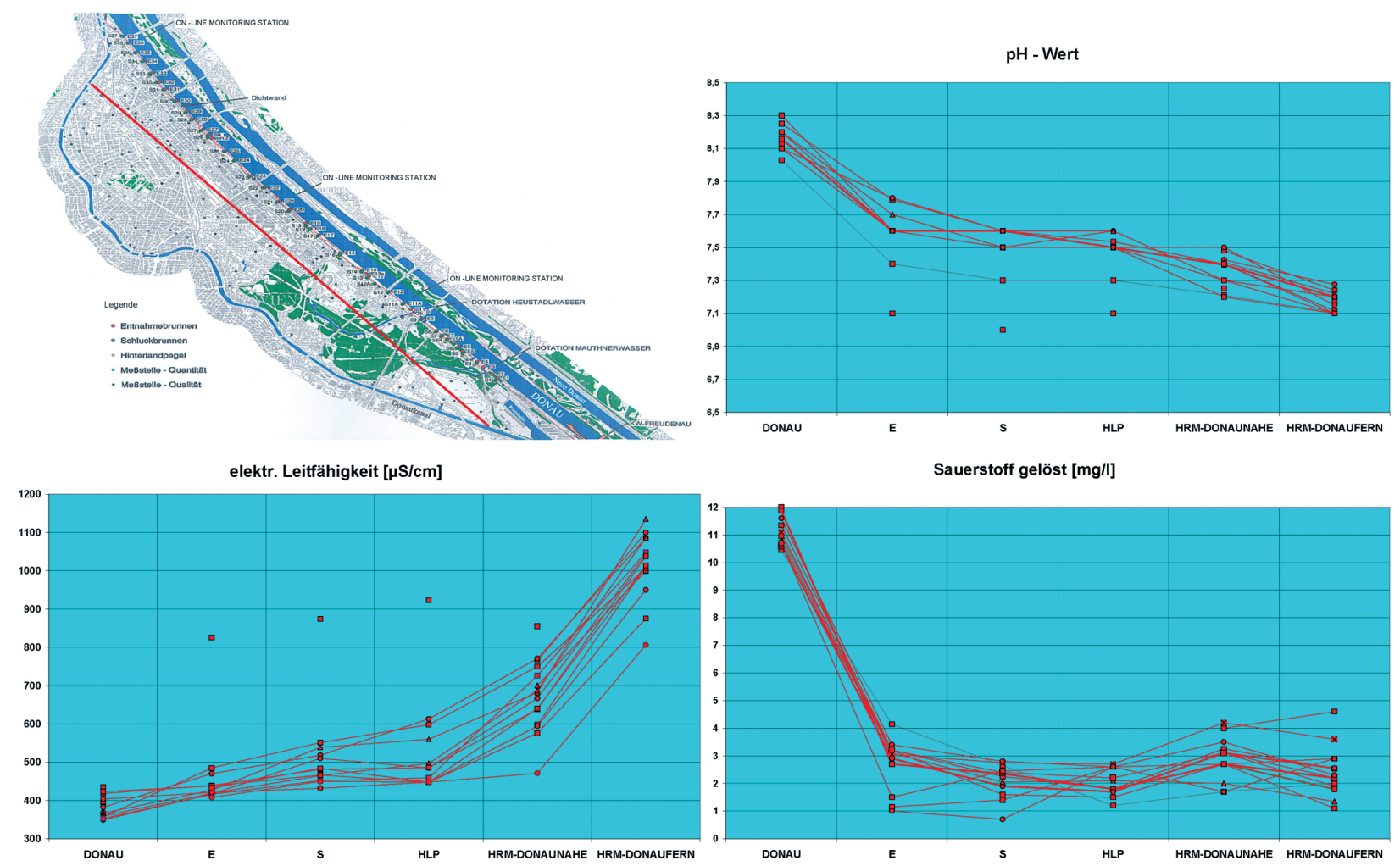

Abb. 3. Änderungen chemisch-physikalischer Parameter im Transekt entlang der Grundwasserfließrichtung „Donau-Hinterland (Donaukanal)“. Es zeigt sich, dass der $\mathrm{pH}$-Wert sukzessive mit zunehmendem Abstand zur Donau abnimmt, wohingegen das Grundwasser sich immer mehr mit lonen anreichert und somit die Leitfähigkeit zunimmt. In den Bewirtschaftungsbrunnen, zwischen Entnahmebrunnen und Schluckbrunnen ist für eine zusätzliche Sauerstoffanreicherung des zur Versickerung gebrachten Uferfiltrates eine Belüftung eingebaut. Diese zeigt jedoch nach langjähriger Beobachtung nur einen geringen bis keinen Erfolg. Der Sauerstoffgehalt nimmt bereits bei der Passage durch den Uferkörper deutlich ab. 
- Bewirtschaftungsmessstellen

- Entnahmebrunnen (E)

- Schluckbrunnen (S)

- Hinterlandpegel (HLP)

- Online-Stationen (OL)

- Beweissicherungsmessstellen

- Hygienisch Relevante Messstellen (HRM)

Spätestens nach Abschluss der Baumaßnahmen zur Dichtwanderrichtung entlang des Donauufers des 2. und 20. Wiener Gemeindebezirkes sowie der Inbetriebnahme der 25 Brunnenanlagen zur Grundwasserbewirtschaftung im Jahre 1996 (Teilstaubeginn) erfolgte eine vollständige Trennung und Entkopplung der natürlich gegebenen Dynamik der Donau und des Aquifers. Somit lassen sich drei voneinander abgrenzbare Zeitbereiche unterscheiden: (1) vor Stauerrichtung („IST-Zustand“) - (2) Zustand während der Stauerrichtung und (3) Endzustand nach Vollstauerrichtung, welche einander gegenüber gestellt und statistisch ausgewertet werden können.

\section{AUSWERTUNGSMETHODE}

Die Ergebnisse der Bewirtschaftungsund Beweissicherungsmessstellen werden laufend auf Grenzwertüberschreitungen bzw. Veränderungen der Wasserqualität untersucht. In jährlichen Bewirtschaftungsberichten werden die Daten zusammengefasst, statistisch ausgewertet, interpretiert und der Behörde vorgelegt (SCHUSTER, 1995-2006). Überschreitungen von Grenzwerten, auffallende Veränderungen wie trendartige Konzentrationszu- bzw. -abnahmen an ein oder mehreren Messstellen sowie plötzliche Veränderungen der Wasserqualität werden durch Nachbeprobungen verifiziert und routinemäßig weiter beobachtet. Basierend aus den Datenauswertungen der Bewirtschaftungsmessstellen lassen sich erste $\mathrm{Zu}$ sammenhänge zwischen Bewirtschaftungsbetrieb und Änderung der Wasserqualität im Nahbereich der Donau herauslesen. Um allfällige Veränderungen der Wasserqualität des Hinterlandes im Zusammenhang mit der Bewirtschaftung aufzuzeigen, wurden jedoch alleinig die Hygienisch relevanten Messstellen (HRM) für weitere Auswertungen herangezogen.

Die Auswertung dieser vorhandenen HRM-Datensätze erfolgte in mehreren Stufen. Im ersten Schritt erfolgte eine Generalaufnahme der vorhandenen Daten - was steht an Parametern (Variablen) zur Verfügung und in

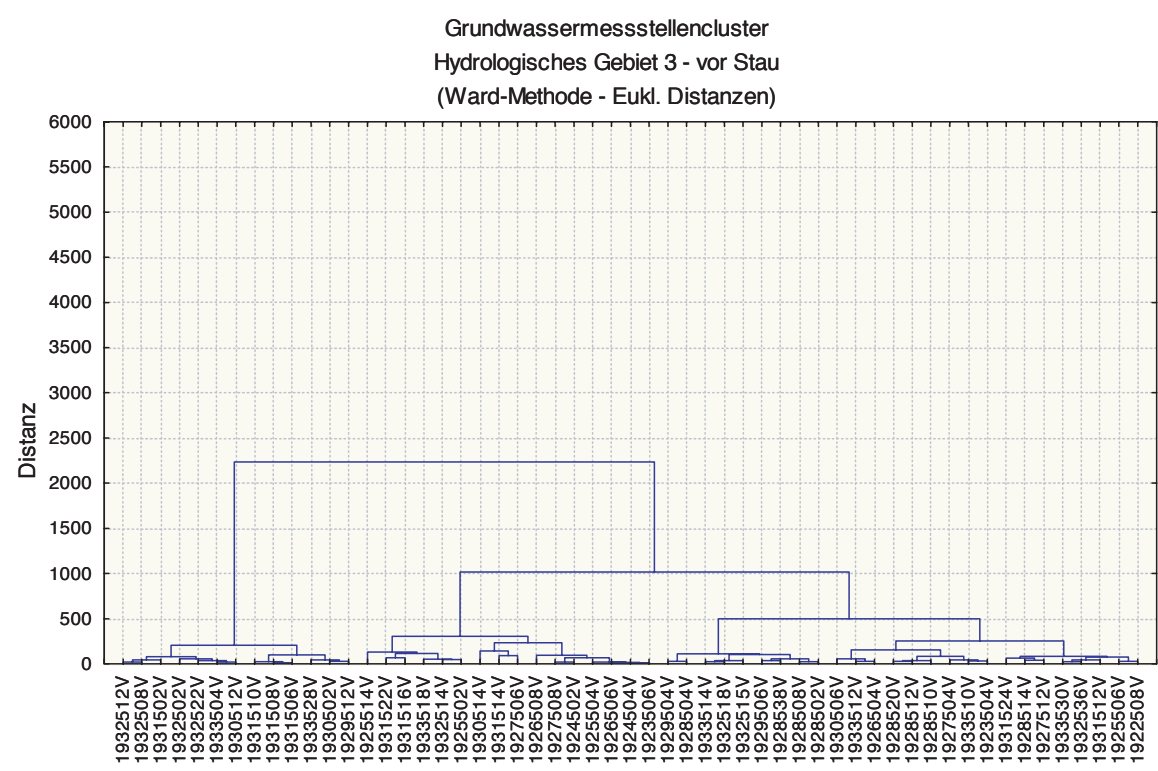

Grundwassermessstellencluster Hydrologisches Gebiet 3 - Vollstau (Ward-Methode - Eukl. Distanzen)

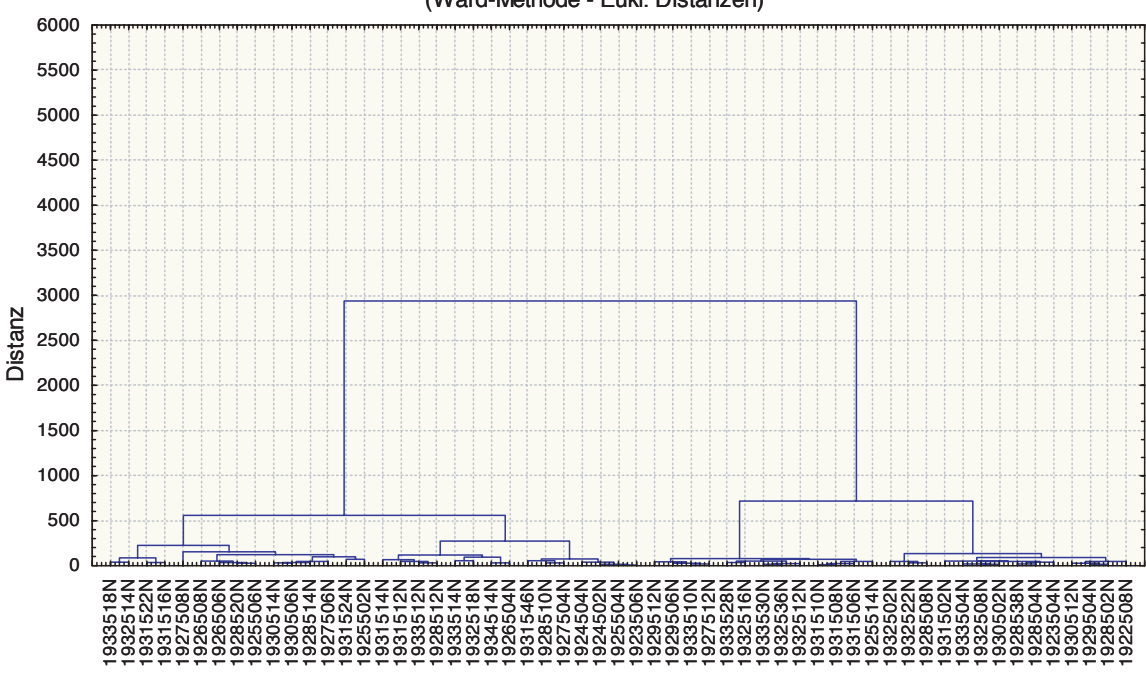

Abb. 4. Messstellenclusteranalyse aller Messdaten der Messstellen des Teilgebietes 2. und 20. Bezirk - vor (oben) und nach Vollstauerrichtung (unten). Veränderungen der Wasserqualität werden durch Änderungen des Ähnlichkeitsverhältnisses der Messstellen zueinander aufgezeigt.

welcher Qualität - sowie eine Bestimmung der deskriptiven Parameter, wie Lagemaße (arithmetisches Mittel, Median) und Streuungsmaße (Standardabweichung, Standardfehler, Spannweite). Aus diesen Parametern ließen sich erste Aussagen über Struktur und Verteilung der Analysewerte machen. Ergebnis dieser Untersuchung war ein Ausschluss nominaler, extrem variationsarmer und inkonsistenter $\mathrm{Da}$ tensätze von bis zu 170 untersuchten Parametern auf zunächst 25 , später 20 Parameter bei Grundwassermessstellen (GW) bzw. 28, später 21 Parameter bei Oberflächenwassermessstellen (OW), welche für eine weitere Analyse verwendet werden, d.h. maximal 300
(GW) bzw. 378 (OW) Korrelationen pro Matrix. Dies war notwendig, da bei einer Vielzahl an untersuchten Parametern, wie z.B. den organischen Spurenelementen (Pestiziden, PCBs, LHKWs), nur Konzentrationen unterhalb der Bestimmungsgrenze gemessen werden konnten.

Im zweiten Schritt erfolgte eine erste Korrelationsanalyse (Spearman) über die Datengesamtheit aller Messstellen, die gepoolten (vor Stau und Vollstau) Einzelmessstellen und über die nach Stauperiode aufgeschlüsselten Einzelmessstellen. Die Spearman Rang-Korrelation diente zur Abrundung des Gesamtbildes, um erste Ideen bezüglich Zusammenhänge 
Tabelle 1. Sperman-Rangkorrelation von 25 Parametern aller Grundwassermessstellen im 2. und 20. Bezirk sowie alle Befunde, gepoolt über die Perioden vor Stau und Vollstau

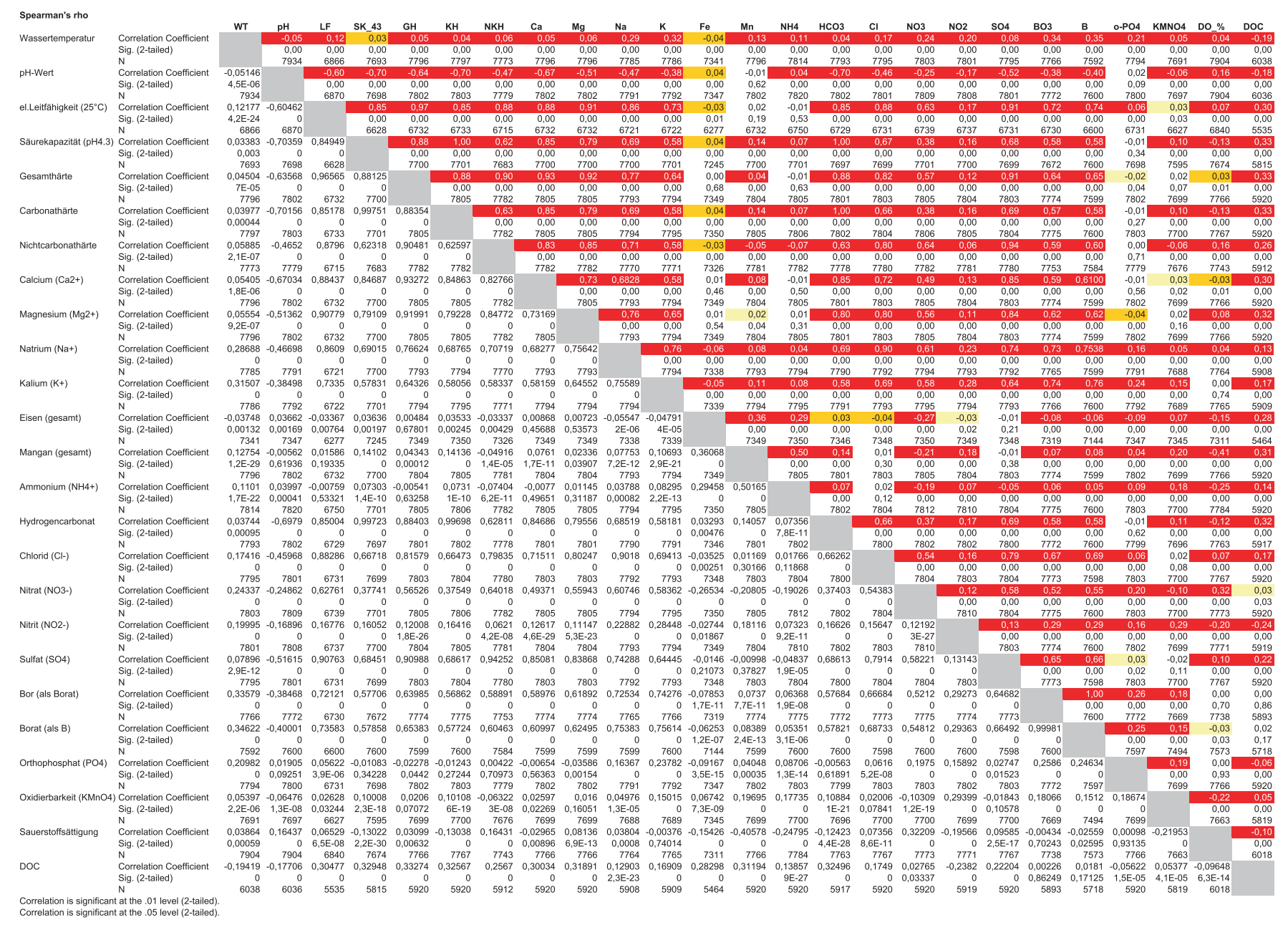

Tabelle 2. Insgesamt wurden über 200 Messstellen im Bereich des 2. und 20. Bezirkes beobachtet. Mehr als 100 verschiedene chemische und bakteriologische Parameter wurden seit 1992 untersucht. Um Änderungen der Grundwasserqualität aufzuzeigen, sind 2 Zeitperioden einander gegenübergestellt. Die Periode vor Stauerrichtung (April 1992 bis März 1996) und die Periode nach Vollstauerrichtung (Jänner 1998 bis Dezember 2006).

\begin{tabular}{|c|c|c|c|c|}
\hline & & $\begin{array}{r}\text { DONAU } \\
1992-2006\end{array}$ & $\begin{array}{r}\text { HINTERLAND } \\
1992-1996\end{array}$ & $\begin{array}{r}\text { HINTERLAND } \\
1998-2006\end{array}$ \\
\hline Wassertemperatur (vor Ort) & ${ }^{\circ} \mathrm{C}$ & 11,4 & 13,3 & 13,2 \\
\hline pH-Wert (Feld) & & 8,2 & 7,23 & 7,2 \\
\hline El. Leitf. $\left(25^{\circ} \mathrm{C}\right)$, Feld & $\mu \mathrm{S} / \mathrm{cm}$ & 370 & 890 & 818 \\
\hline Gesamthärte & ${ }^{\circ} \mathrm{dH}$ & 11 & 23,1 & 21,2 \\
\hline Calcium $\left(\mathrm{Ca}^{2+}\right)$ & $\mathrm{mg} / \mathrm{l}$ & 58 & 120 & 105,4 \\
\hline Magnesium $\left(\mathrm{Mg}^{2+}\right)$ & $\mathrm{mg} / \mathrm{l}$ & 12 & 27 & 28,4 \\
\hline Natrium (gelöst) $\left(\mathrm{Na}^{+}\right)$ & $\mathrm{mg} / \mathrm{l}$ & 8,6 & 25 & 24,3 \\
\hline Kalium (gelöst) $\left(K^{+}\right)$ & $\mathrm{mg} / \mathrm{l}$ & 2,4 & 5 & 5,3 \\
\hline Hydrogencarbonat $\left(\mathrm{HCO}_{3}{ }^{-}\right)$ & $\mathrm{mg} / \mathrm{l}$ & 193 & 380 & 350 \\
\hline Chlorid $\left(\mathrm{Cl}^{-}\right)$ & $\mathrm{mg} / \mathrm{l}$ & 14,8 & 40 & 37,55 \\
\hline Nitrat $\left(\mathrm{NO}_{3}{ }^{-}\right)$ & $\mathrm{mg} / \mathrm{l}$ & 9,6 & 24 & 18,5 \\
\hline Sulfat $\left(\mathrm{SO}_{4}{ }^{2-}\right)$ & $\mathrm{mg} / \mathrm{l}$ & 26,4 & 81,85 & 76,2 \\
\hline Orthophosphat $\left(\mathrm{OPO}_{4}^{3-}\right)$ & $\mathrm{mg} / \mathrm{l}$ & 0,108 & 0,046 & 0,05 \\
\hline Phosphat gesamt gelöst $\left(\mathrm{PO}_{4}{ }^{3-}\right)$ & $\mathrm{mg} / \mathrm{l}$ & 0,162 & 0,07 & 0,09 \\
\hline Sauerstoff, gelöst $\left(\mathrm{O}_{2}\right)$ & $\mathrm{mg} / \mathrm{l}$ & 11 & 2,2 & 2,8 \\
\hline DOC (Dissolved organic carbon) & $\mathrm{mg} / \mathrm{l}$ & 2,655 & 0,5 & 0,75 \\
\hline Aluminium, gelöst & $\mathrm{mg} / \mathrm{l}$ & 0,038 & $<0,01$ & $<0,01$ \\
\hline Aer,heterotr,Keime $\left(22^{\circ} \mathrm{C} / 48 \mathrm{~h}\right)$ & $\mathrm{KBE} / \mathrm{ml}$ & 1190 & 20 & 30,5 \\
\hline Aer,heterotr,Keime $\left(37^{\circ} \mathrm{C} / 48 \mathrm{~h}\right)$ & $\mathrm{KBE} / \mathrm{ml}$ & 163 & 1 & 15 \\
\hline Escherichia coli & $1 / 100 \mathrm{ml}$ & 64 & 0 & 0 \\
\hline Enterokokken (Fäkalstreptokokken) & $1 / 100 \mathrm{ml}$ & 50 & 0 & 0 \\
\hline sulfitreduzierende Clostridien & $1 / 20 \mathrm{ml}$ & 1 & 0 & 0 \\
\hline Pseudomonas aeruginosa & $1 / 100 \mathrm{ml}$ & 5,5 & 0 & 0 \\
\hline
\end{tabular}

zu erhalten. Diese Rangkorrelation rechnet nicht mit den Zahlen selbst, sondern mit deren Rängen - eine Normalverteilung ist deshalb nicht notwendig. Das Ergebnis liefert einen Korrelationskoeffizienten $|\rho|$ als $\mathrm{Zu}$ sammenhangsmaß. Je größer der $\mathrm{Zu}$ sammenhang ist, desto näher liegt $|\rho|$ bei 1, gibt es keinen Zusammenhang, so liegt $|\rho|$ bei 0 . Abschließend wird ein statistischer Test durchgeführt, dessen Nullhypothese lautet: der Korrelationskoeffizient ist gleich 0 (also kein Zusammenhang zwischen den beiden analysierten Variablen!).

Für alle statistischen Tests werden drei Signifikanzniveaus verwendet: $\alpha=0,001$ für höchst signifikant, $a=0,01$ für hoch signifikant und $\alpha=0,05$ für signifikant. Fehlerwahrscheinlichkeiten $>0,05$ werden als nicht signifikant bezeichnet, was bedeutet, dass die Nullhypothese nicht verworfen werden kann.

In den Ergebnissen der SpermanRangkorrelation aller 25 Grundwasser-Parameter wurde der Grad der 
Tabelle 3. Detailauswertung der Beispiel-Messstelle 1930,514 - Lasalleestraße. Neben Mittelwert pro Untersuchungszeitraum wurden erweiterte statistische Kenngrößen miterfasst. Signifikante Änderungen pro Parameter wurden in der letzten Spalte farblich rot markiert, wobei eine Konzentrationszu- oder -abnahme in den Spalten mit > oder < dargestellt wurden.

\begin{tabular}{|c|c|c|c|c|c|c|c|c|c|c|}
\hline & $\begin{array}{r}\text { Stationsnummer } \\
\text { Stationsname } \\
\text { Ablagenummer } \\
\text { Postzahl }\end{array}$ & $\begin{array}{l}\text { 1930_514 } \\
\text { Bank Aus } \\
278 \\
3279\end{array}$ & ria - Lasall & traße & & & & & & \\
\hline \multirow[b]{2}{*}{ Nr. } & \multirow[b]{2}{*}{ Parameterbezeichnung } & \multirow[b]{2}{*}{ Par. Dim. } & \multirow{2}{*}{\begin{tabular}{|c|} 
vor Stau \\
$\begin{array}{c}\text { Mittel- } \\
\text { wert }\end{array}$
\end{tabular}} & \multirow{2}{*}{\begin{tabular}{|c|} 
Vollstau \\
$\begin{array}{c}\text { Mittel- } \\
\text { wert }\end{array}$ \\
\end{tabular}} & \multirow[b]{2}{*}{ Kommentar } & \multirow[b]{2}{*}{$\begin{array}{l}\text { F-Test } \\
\text { (Varianz) }\end{array}$} & \multicolumn{2}{|c|}{ bei Vollstau } & \multirow[b]{2}{*}{$\begin{array}{l}\text { t-Test } \\
(p)\end{array}$} & \multirow[b]{2}{*}{$\begin{array}{l}\text { Sign. } \\
\text { Untersch }\end{array}$} \\
\hline & & & & & & & Mittelwert & Median & & \\
\hline & $\begin{array}{l}\text { A.) CHEM.PHYS.STANDARDUNTERSUCHUNG } \\
\text { physikalische Parameter }\end{array}$ & & & & & & & & & \\
\hline 3102 & Lufttemperatur & ${ }^{\circ} \mathrm{C}$ & 4,167 & 14,69 & t-Test & homogen & $>$ & $>$ & 0,008 & JA \\
\hline 3101 & Wassertemperatur (vor Ort) & ${ }^{\circ} \mathrm{C}$ & 17,6 & 16,12 & t-Test & homogen & $<$ & $<$ & 0,021 & JA \\
\hline 3070 & pH-Wert (Ort und Stelle) & & 7,003 & 6,975 & $\mathrm{t}$-Test & homogen & $<$ & $<$ & 0,572 & NEIN \\
\hline 3071 & pH-Wert (Labor) & & & & Anz. $=0$ & no $F$ & & & & \\
\hline 3076 & Redoxpotential (Ort und Stelle) & $\mathrm{mV}$ & & 405,6 & Anz. $=0$ & no $F$ & & & & \\
\hline 3095 & Spekt. Abs. Koeff.: bei $254 \mathrm{~nm}$ filtriert & $1 / \mathrm{m}$ & & 2,482 & Anz. $=0$ & no F & & & & \\
\hline 3096 & Spekt. Abs. Koeff.: bei $436 \mathrm{~nm}$ filtriert & $1 / \mathrm{m}$ & & 0,104 & Anz. $=0$ & no F & & & & \\
\hline 3013 & el.Leiffähigkeit $\left(25^{\circ} \mathrm{C}\right.$, Ort und Stelle) & $\mu \mathrm{S} / \mathrm{cm}$ & & 1083 & Anz. $=0$ & no F & & & & \\
\hline 3014 & el.Leitfähigkeit $\left(25^{\circ} \mathrm{C}\right.$, Labor $)$ & $\mu \mathrm{S} / \mathrm{cm}$ & & & Anz. $=0$ & no $F$ & & & & \\
\hline 3015 & el.Leitfähigkeit $\left(20^{\circ} \mathrm{C}\right.$, Ort und Stelle) & $\mu \mathrm{S} / \mathrm{cm}$ & & 920,4 & Anz. $=0$ & no $F$ & & & & \\
\hline \multirow[t]{2}{*}{3016} & el.Leitfähigkeit $\left(20^{\circ} \mathrm{C}\right.$, Labor $)$ & $\mu \mathrm{S} / \mathrm{cm}$ & 1383 & & Anz. $=0$ & no $F$ & & & & \\
\hline & Wasserhärte & & & & & & & & & \\
\hline 2202 & Säurekapazität (pH4.3) & $\mathrm{mmol} / \mathrm{I}$ & 9,25 & 7,202 & t-Test & heterogen & $<$ & $<$ & 0,000 & JA \\
\hline 2286 & Gesamthärte & ${ }^{\circ} \mathrm{dH}$ & 35,12 & 27,12 & t-Test & homogen & $<$ & $<$ & 0,000 & JA \\
\hline 2288 & Carbonathärte & ${ }^{\circ} \mathrm{dH}$ & 25,85 & 20,18 & t-Test & heterogen & $<$ & $<$ & 0,000 & JA \\
\hline \multirow[t]{2}{*}{2290} & Nichtcarbonathärte & ${ }^{\circ} \mathrm{dH}$ & 9,267 & 6,93 & t-Test & heterogen & $<$ & $<$ & 0,087 & NEIN \\
\hline & Kationen & & & & & & & & & \\
\hline 2273 & Calcium $(\mathrm{Ca} 2+)$ & $\mathrm{mg} / \mathrm{l}$ & 166,7 & 133 & t-Test & homogen & $<$ & $<$ & 0,000 & JA \\
\hline 2298 & Magnesium (Mg2+) & $\mathrm{mg} / \mathrm{l}$ & 51,5 & 36,87 & t-Test & homogen & $<$ & $<$ & 0,000 & JA \\
\hline 2304 & Natrium $(\mathrm{Na}+)$ & $\mathrm{mg} / \mathrm{l}$ & 58,67 & 37,19 & t-Test & heterogen & $<$ & $<$ & 0,000 & JA \\
\hline 2294 & Kalium $(\mathrm{K}+)$ & $\mathrm{mg} / \mathrm{l}$ & 11,83 & 10,52 & $\mathrm{t}$-Test & homogen & $<$ & $<$ & 0,021 & JA \\
\hline 2283 & Eisen (gelöst) & $\mathrm{mg} / \mathrm{l}$ & & 0,04038 & Anz. $=0$ & no $F$ & & & & \\
\hline 2284 & Eisen (gesamt) & $\mathrm{mg} / \mathrm{l}$ & 0,01167 & 0,01125 & $\mathrm{t}$-Test & homogen & $<$ & $=$ & 0,856 & NEIN \\
\hline 2281 & Eisen II & $\mathrm{mg} / \mathrm{l}$ & & 0,028 & Anz. $=0$ & no $F$ & & & & \\
\hline 2537 & Mangan (gesamt) & $\mathrm{mg} / \mathrm{l}$ & 0,1583 & 0,05885 & $\mathrm{t}$-Test & homogen & $<$ & $<$ & 0,000 & JA \\
\hline 2565 & Mangan II & $\mathrm{mg} / \mathrm{l}$ & & 0,04875 & Anz. $=0$ & no $F$ & & & & \\
\hline \multirow[t]{2}{*}{2309} & Ammonium (NH4+) & $\mathrm{mg} / \mathrm{l}$ & 0,2162 & 0,0335 & $\mathrm{t}$-Test & heterogen & $<$ & $<$ & 0,000 & JA \\
\hline & Anionen & & & & & & & & & \\
\hline 2291 & Hydrogencarbonat & $\mathrm{mg} / \mathrm{l}$ & 563,3 & 440,1 & t-Test & heterogen & $<$ & $<$ & 0,000 & JA \\
\hline 2318 & Chlorid (Cl-) & $\mathrm{mg} / \mathrm{l}$ & 79,67 & 51,29 & t-Test & homogen & $<$ & $<$ & 0,000 & JA \\
\hline 2333 & Nitrat (NO3-) & $\mathrm{mg} / \mathrm{l}$ & 26,67 & 15,2 & t-Test & homogen & $<$ & $<$ & 0,000 & JA \\
\hline 2336 & Nitrit (NO2-) & $\mathrm{mg} / \mathrm{l}$ & 0,02067 & 0,0277 & t-Test & homogen & $>$ & $>$ & 0,307 & NEIN \\
\hline 2343 & Sulfat (SO4) & $\mathrm{mg} / \mathrm{l}$ & 205 & 134,7 & t-Test & homogen & $<$ & $<$ & 0,000 & JA \\
\hline 2310 & Bor (als Borat) & $\mathrm{mg} / \mathrm{l}$ & 1,07 & 0,9188 & t-Test & homogen & $<$ & $<$ & 0,077 & NEIN \\
\hline 2311 & Borat (als B) & $\mathrm{mg} / \mathrm{l}$ & 0,1967 & 0,1688 & t-Test & homogen & $<$ & $<$ & 0,075 & NEIN \\
\hline 2213 & Gesamtphosphat (als PO4) & $\mathrm{mg} / \mathrm{l}$ & & 0,0996 & Anz. $=0$ & no $F$ & & & & \\
\hline 2215 & Orthophosphat (PO4) & $\mathrm{mg} / \mathrm{l}$ & 0,02683 & 0,05265 & t-Test & heterogen & $>$ & $>$ & 0,023 & JA \\
\hline 2216 & Orthophosphat (als PO4-P) & $\mathrm{mg} / \mathrm{l}$ & & & Anz. $=0$ & no $F$ & & & & \\
\hline 2212 & Gesamtphosphat (als PO4) & $\mathrm{mg} / \mathrm{l}$ & & & Anz. $=0$ & no F & & & & \\
\hline \multirow[t]{2}{*}{2217} & Phosphor, gesamt & $\mathrm{mg} / \mathrm{l}$ & & & Anz. $=0$ & no $F$ & & & & \\
\hline & Sauerstoff und org. Summenparameter & & & & & & & & & \\
\hline 2030 & Oxidierbarkeit (KMnO4-verbr.) & $\mathrm{mg} / \mathrm{l}$ & 1,029 & 1,1 & t-Test & homogen & $>$ & $<$ & 0,594 & NEIN \\
\hline 3078 & Sauerstoffgehalt (elektrometr.) & $\mathrm{mg} / \mathrm{l}$ & 1,567 & 2,15 & t-Test & homogen & $>$ & $>$ & 0,402 & NEIN \\
\hline 3080 & Sauerstoffsättigung & $\%$ & 17,5 & 22,14 & t-Test & homogen & $>$ & $>$ & 0,527 & NEIN \\
\hline 1619 & Total organic carbon (TOC) & $\mathrm{mg} / \mathrm{l}$ & 1,556 & 1,313 & $\mathrm{t}$-Test & homogen & $<$ & $<$ & 0,461 & NEIN \\
\hline 2016 & Dissolved organic carbon (DOC) & $\mathrm{mg} / \mathrm{l}$ & & 1,191 & Anz. $=0$ & no $F$ & & & & \\
\hline
\end{tabular}

Korrelation in unterschiedlicher Farbe unterlegt. So sind höchst signifikante Niveaus $[\alpha=0,001]$ rot unterlegt, hoch signifikante Niveaus $[\alpha=0,01]$ orange und signifikante Niveaus $[\alpha=0,05]$ gelb. Fehlerwahrscheinlichkeiten $>0,05$ werden als nicht signifikant bezeichnet und sind nicht farblich unterlegt.

In einem dritten Schritt wurden die Ähnlichkeiten zwischen den 20 (GW) bzw. 21 (OW) Parametern mittels Clusteranalyse untersucht und so die in der Korrelationsanalyse gefundenen Muster bestätigt (siehe Abb. 1 und 2).

Für die Grundwassermessstellen zeigt sich, dass generell 2 Hauptcluster unterschieden werden können. Der erste Cluster beinhaltet Leitfähigkeit und Härte dominierende Bestandteile, wie die Hauptkationen und Anionen.
Wobei die Gesamthärte am stärksten mit der Leitfähigkeit sowie naturgemäß mit Kalzium und Magnesium korreliert. Nichtkarbonathärte wird vor allem durch Sulfat bestimmt, mit einem Korrelationskoeffizienten von 0,94 . Natrium steht am deutlichsten mit Chlorid bei einem Korrelationskoeffizienten von 0,9 in Zusammenhang. In dem zweiten Hauptcluster finden sich neben chemisch-physikalischen Parametern, wie Wassertemperatur, $\mathrm{pH}$-Wert und Sauerstoffsättigung, auch Phosphat, Kalium, Nitrat sowie DOC und Oxidierbarkeit (Permanganatverbrauch) wieder. Die Wassertemperatur steht meist abgesetzt von den anderen Parametern, ist leicht assoziiert mit Orthophosphat bzw. mit Kalium und Nitrat.

Ähnliches findet sich bei den Ober- flächenwassermessstellen. Deutlich zu sehen ist, dass auch hier zwei Hauptcluster unterschieden werden können. Wie bei den Grundwassermessstellen beinhaltet der erste Hauptcluster vorwiegende Hauptkationen und -anionen, während der zweite Cluster organische Belastungsindikatoren, wie $\mathrm{KMnO} 4$, DOC, BSB5 und Phosphate, enthält sowie pH-Wert und Sauerstoffgehalt. Auch bei den Oberflächenwassermessstellen zeigt Sauerstoff eine höchste Korrelation mit dem pH-Wert $(0,49)$. BSB5 steht am deutlichsten mit DOC sowie dem Permanganatverbrauch im Zusammenhang. Die Wassertemperatur steht meist abgesetzt von den anderen Parametern, ist leicht assoziiert mit Gesamthärte und Nichtkarbonathärteparametern. 

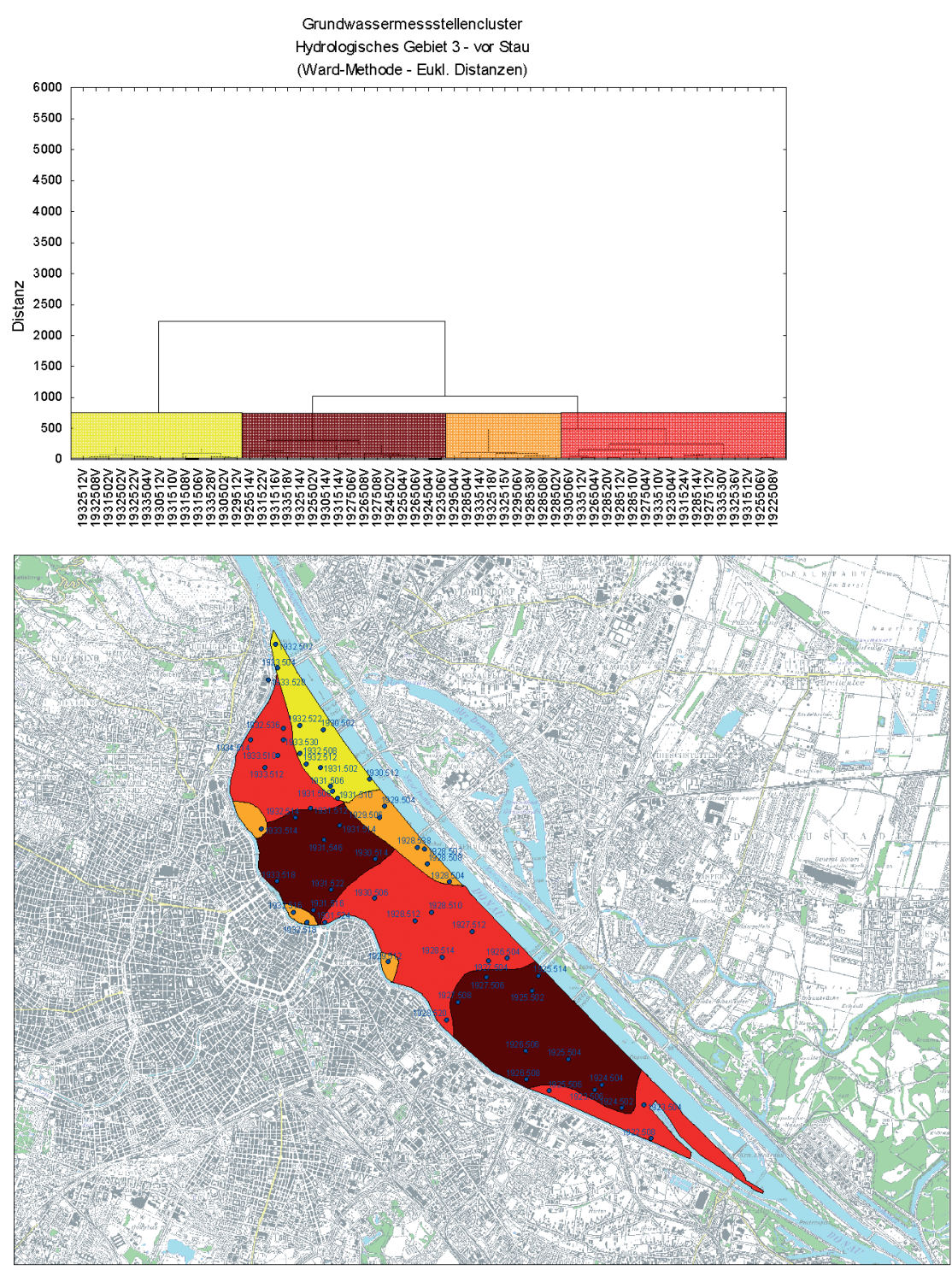

Abb. 5. Zusammenfassung der einzelnen Messstellencluster (Ähnlichkeitsdistanz >500) vor Stau zu farblich unterlegten regionalen Gruppen. Abgegrenzt die Messstellen des gelben Clusters, welche allesamt im nördlichen Teil entlang der Donau bzw. des Altarmes in diesem Gebiet liegen.
In einem vierten und letzten Schritt wurden die Untersuchungsergebnisse jener Messstellen mit signifikanten Zunahmen bzw. Abnahmen der mittleren Konzentrationen bzw. des Median pro Zeitperiode einzelner Parameter für eine weitere Beurteilung herangezogen. Basierend auf den Messdaten und den Untersuchungsergebnissen erfolgte eine Gesamtbeurteilung hinsichtlich einer möglichen chemischen Veränderung der Grundwasserqualität im hydrologischen Randgebiet.

\section{ERGEBNISSE}

Anhand des langjährigen Beobachtungszeitraumes kann aufgrund der Nähe zur Donau ein Oberflächenwas- ser-beeinflusster Charakter des Uferfiltrats festgestellt werden. Der Donaueinfluss lässt sich je nach Brunnen, abhängig von Pumpleistung des Brunnens bzw. Kolmationsgrad der Uferbereiche, mehr oder weniger deutlich an den Wasserqualitätsdaten erkennen. Im Vergleich zu den Entnahmebrunnen zeigt sich, dass die Hinterlandpegel einem deutlicheren Einfluss des Hinterlandes unterliegen. Dies bedeutet, dass aufgrund der Grundwasserbewirtschaftung für den 2. und 20. Bezirk der Grundwasserkörper durch die Dotationsmaßnahmen, bei denen Uferfiltrat aus dem Uferbereich der Donau entnommen und jenseits der Dichtwand im Hinterland über die Schluckbrunnen ver- sickert wird, eine „Verdünnung“ der gelösten Stoffe erfolgt und somit $\mathrm{Pa}$ rameter wie Leitfähigkeit und Härten (Kationen und Anionen) Donaucharakter annehmen. Im Gegensatz dazu wird während der Rückpumpphasen der Brunnenanlagen dem Grundwasserkörper Wasser entnommen und in die Donau gepumpt. Dadurch kommt es zu einer Änderung der Grundwasserfließrichtung, bei denen die Hinterlandpegel vom ionenreichen Grundwasser des Hinterlandes des 2. und 20. Bezirks bzw. durch den Donaukanal beeinflusst werden, weshalb die Konzentration an Ionen deutlich ansteigen (siehe Abb. 3) (SCHUSTER und PICHER, 2006).

Änderungen der chemischen Grundwasserqualität im Lokalbereich der Messstelle selbst bzw. großflächig im gesamten hydrologischen Gebiet können durch Clusteranalysen und zusätzliche detaillierte Einzelbeurteilungen der Messstellen aufgezeigt werden. Pro Beobachtungsperiode, d.h. vor Vollstauerrichtung und nach Vollstauerrichtung wurden die Messstellen zueinander korreliert, um Ähnlichkeiten bzw. Veränderungen der Messstellenassoziationen aufzuzeigen (siehe Abb. 4).

Diese Auswertung zeigt Ähnlichkeiten der Messstellen in den Perioden vor Stau sowie nach Vollstauerrichtung zueinander an und lässt erkennen, wie Grundwasserverhältnisse im Untersuchungsgebiet großflächig verteilt sind. Änderungen der Wasserqualität an den Messstellen selbst sowie im Zusammenhang zu benachbarten Messstellen können durch eine Clusteranalyse sämtlicher Messdaten der Perioden vor Stau sowie nach Vollstau ausfindig gemacht werden. Treten keine Veränderungen in der Grundwasserqualität auf, so zeigt sich dies daran, dass einzelne Messstellen sich selbst am ähnlichsten sind und somit nebeneinander in den Messstellenclusterdiagrammen zu liegen kommen.

Anhand der Messstellenclusteranalysen ist ersichtlich, dass im Gesamtpool aller Analysewerte der Messstellen vor und nach Vollstau deutlich 2 Haupt- und 4 Nebencluster unterschieden werden können. Auffallend sind bei dieser Darstellung, die Unterschiede bzw. Ähnlichkeiten der einzelnen Messstellen zeitlich zueinander (vor Stau zu Vollstau) bzw. zwischen den Messstellen. Es zeigt sich, dass manche Messstellen sich selbst am ähnlichsten sind (kaum eine Veränderung der Wasserqualität), wohingegen andere Messstellen eine deutliche Änderung der Hauptclusterzugehörigkeit 
vor Stau und Vollstau aufzeigen (große Veränderung der Wasserqualität). Der Großteil der untersuchten Messstellen behält die Ähnlichkeit im jeweiligen Hauptcluster bei, was einer geringfügigen Änderung der Dynamik bzw. einer geringen Konzentrationsänderung der berücksichtigten Parameter der Messstellen des Teilclusters entspricht. Die Ergebnisse der Clusteranalyse vor Stau und nach Vollstau wurden in den Messstellenplänen grafisch dargestellt.

Während die Clusteranalyse aller Messstellen, basierend auf den statistisch ausgewerteten Messparametern, eine Ähnlichkeit der Messstellen zueinander aufzeigt, gibt diese Auswertung und Art der Darstellung keine Information über tatsächliche chemische Veränderungen und deren Intensität im Untersuchungsgebiet selbst wieder. Hierfür ist eine vertiefte Auswertung der chemischen Untersuchungsergebnisse aller Messstellen der Gesamtperiode vor Stau und nach Vollstauerrichtung zusätzlich notwendig. Hierfür wurden von allen 56 Grundwassermessstellen insgesamt 2125 Befunde mit 143496 Einzelmesswerten ausgewertet. In Tabelle 2 sind für einzelne Parameter die Mittelwerte der Donaumessstellen für die Periode 1992-2006 sowie für die Messstellen im Hinterland die beiden Perioden 1992-1996 (vor Stau) und 1998-2006 (nach Vollstauerrichtung) einander gegenübergestellt.

Eine Tendenz zur Verringerung der Leitfähigkeit $(\approx 70 \mu \mathrm{S} / \mathrm{cm})$, Härten $\left(\approx 2^{\circ} \mathrm{dH}\right)$, Hauptkationen und Anionen kann im gesamten Untersuchungsgebiet beobachtet werden. Gleichzeitig ist eine leichte Zunahme der Wassertemperatur, des pH-Wertes im Nahbereich der Donau sowie der Oxidierbarkeit, des DOC-Gehaltes und der Sauerstoffsättigung zu verzeichnen. Diese Trends pro Hauptcluster, visualisiert in einem Lageplan, lassen deutlich den unterschiedlich abgrenzbaren Bereich nahe der Donau sowie jene weiter im Hinterland des 2. und 20. Bezirkes gelegenen aufzeigen. Bei den Messstellen im Nahbereich der Donau ist im Vergleich zu den Hinterlandmessstellen eine Verringerung der Natrium-, Kalium-, Bor- und Orthophosphat-Konzentration zu verzeichnen, wohingegen die Hinterlandmessstellen eine geringfügige $\mathrm{Zu}$ nahme aufweisen.

Durch den Einfluss der Grundwasserbewirtschaftung des 2 . und 20. Bezirkes konnte eine Verdünnung des donaunahen Grundwassers erreicht werden. Die in der Mitte des
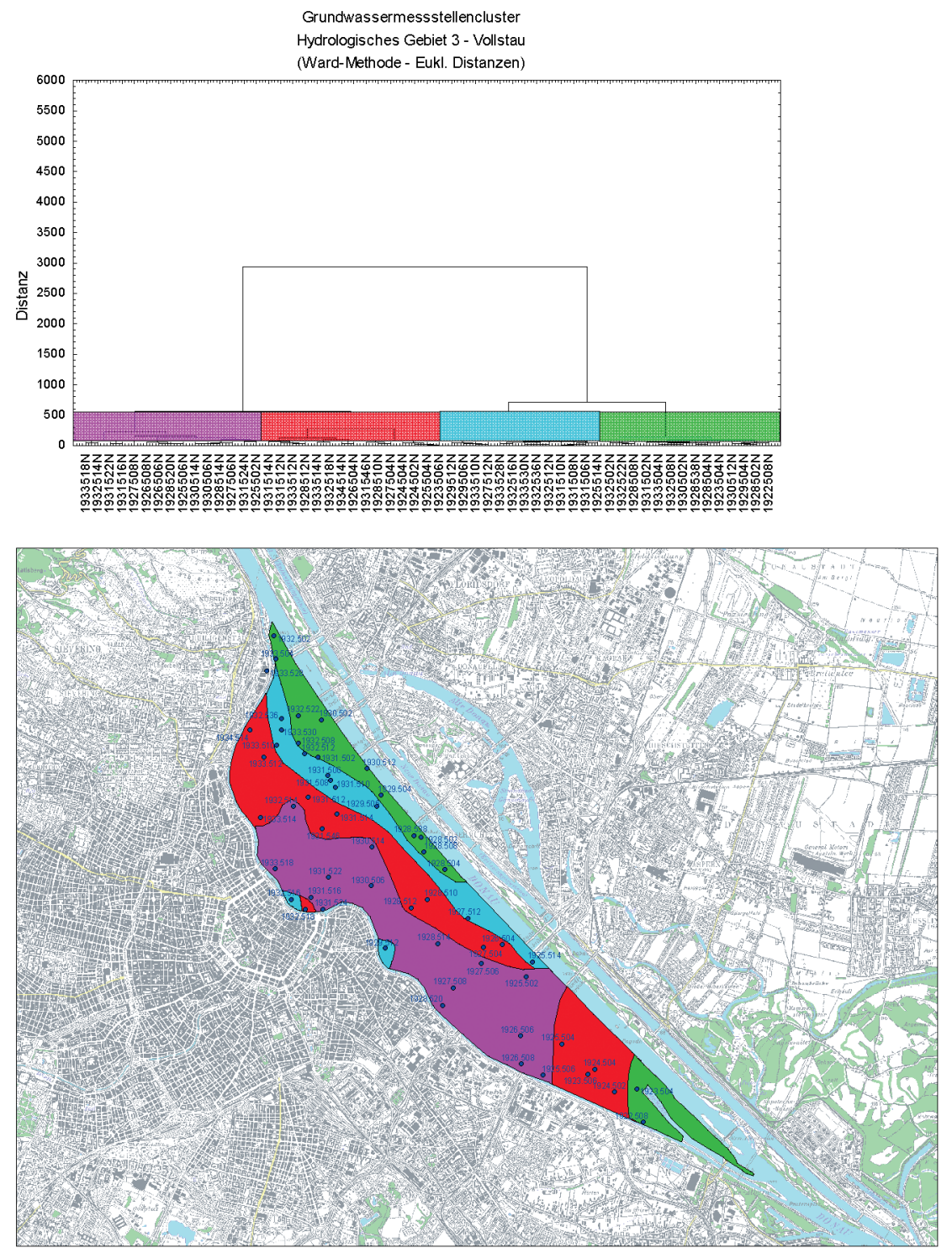

Abb. 6. Zusammenfassung der einzelnen Messstellencluster (Ähnlichkeitsdistanz > 500) nach Vollstau zu farblich unterlegten regionalen Gruppen. Die beiden Hauptclustergruppen grün und hellblau liegen allesamt in den durch die Bewirtschaftung beeinflussten Donaunahen gebieten. Die Veränderung der Wasserqualität breitet sich „bandförmig“ ins Hinterland aus.

2. und 20. Bezirkes gelegenen Messstellen weisen von Natur aus eine deutlich höhere Ionenkonzentration auf, welche durch die Grundwasserbewirtschaftung nur geringfügig und langsam verändert werden konnte. Es zeigt sich außerdem, dass durch die natürliche Filterwirkung des Uferbereiches, indem die Entnahmebrunnen Uferfiltrat fördern und nicht direkt Wasser aus der Donau entnehmen, Keime bzw. Viren großteils herausgefiltert werden und es somit zu keiner bakteriologischen Verunreinigung kommen kann.

Neben einer Gesamtbeurteilung des untersuchten Gebietes erfolgte weiters eine vertiefte Untersuchung pro Messstelle und Parameter. Sämt- liche Analysenergebnisse der Perioden vor Stau und nach Vollstauerrichtung wurden mit Unterstützung einer eigens entwickelten Datenbank und weiterer digitaler Datenverarbeitung tabellarisch zusammengefasst (Tabelle 3).

Hierbei zeigt sich, ob der Mittelwert bzw. Median eines Parameters im Vergleich der beiden Zeitbereiche eine signifikante Zunahme oder Abnahme bzw. keinerlei Veränderung aufweist. Zur weiteren Verdeutlichung von Veränderungen der Wasserqualität an der Messstelle selbst wurden die Mittelwerte vordefinierter Parameter pro Untersuchungsperiode (vor Stau bzw. nach Vollstau) als Starplot dargestellt (siehe Abb. 7). 

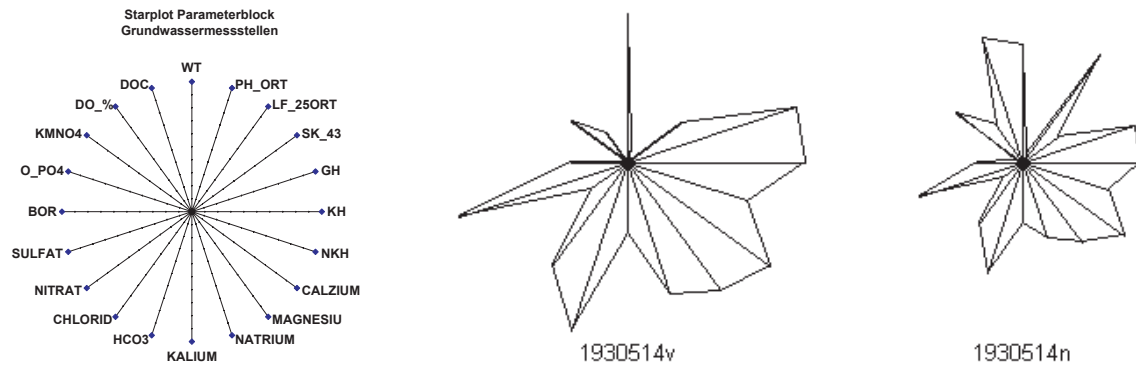

Abb. 7. Verteilung ausgewählter chemischer Wasserqualitätsparameter der Grundwassermessstellen im Uhrzeigersinn und Visueller Direktvergleich der Analysenergebnisse der Messstelle 1930,514 vor Stau und nach Vollstau am Beispiel einer Starplot Darstellung. Hohe mittlere Konzentrationen bzw. Messwerte sind durch lange Strahlen gegeben. Der direkte Vergleich beider „Sterne“ zeigt die Zunahme bzw. Abnahme der mittleren Konzentration eines Parameters an.

Die hier ausgewählten 20 Parameter sollen einen Überblick über die möglichen Veränderungen der Matrix der Gewässer aufzeigen. Sie lassen sich in zwei Gruppen zusammenfassen: $\mathrm{Pa}-$ rameter als Einzugsgebiet-Indikatoren wie pH-Wert, Leitfähigkeit, Gesamthärte, Nichtcarbonathärte, Calcium, Kalium und Hydrogencarbonat (Position 0-7 Uhr), Parameter für Anzeiger anthropogener Aktivitäten wie Chlorid, Nitrat, Bor, Phosphat, gelöster Sauerstoff (DO) und gelöster organischer Kohlenstoff (DOC) (Position 7-12 Uhr). Dabei kann man grob zwischen jenen Parametern unterscheiden, welche bedingt durch den Untergrund „von Natur aus" vorkommen und somit den Typus des Wassers bestimmen, und jenen Substanzen, welche zusätzlich durch autogene und/oder allogene Beeinträchtigungen unter Umständen sogar bis in den toxischen Bereich erhöht sein können. Demzufolge werden in der Gruppe der Einzugsgebietesindikatoren die untersuchten Parameter Leitfähigkeit (als Maß für die im Wasser enthaltenen und gelösten Ionen), Gesamthärte (als Maß für die im Wasser enthaltenen Kalzium, Magnesium, Strontium und Barium-Ionen), Nichtkarbonathärte (jener Anteil der Gesamthärte, welcher nicht durch die Auflösung von Karbonaten, sondern z.B. aus Sulfaten, Chloriden, Nitraten, Silikaten und Phosphaten stammt), Kalzium (als Hauptkation), Kalium (ein Indikator für mögliche Düngemittelauswaschungen in humusarmen Böden infolge von Niederschlägen) und Hydrogenkarbonat (auch als Maß für die Pufferkapazität des Wassers) zusammengefasst.
Des Weiteren sind die untersuchten Parameter Chlorid (als Indikator von Verunreinigungen durch Abwässer, Straßenstreusalz oder z.B. Kalidünger (KCl), Nitrat (als Indikator für Düngerauswaschungen sowie Abbau- und Oxidationsvorgängen organischer und anorganischer Stoffe), Bor (als Indikator von Bor-Verunreinigungen aus Email- und Glasindustrien, Galvanik, Photochemie, Mineralfarbenherstellungsbetrieben sowie aus Imprägnierungsbetrieben), Phosphat (Indikator für Abwässer, Düngemittel und Waschmittel) sowie gelöster Sauerstoff und organischer Kohlenstoff (als Indikatoren für anthropogene Altlasten) in der Gruppe der Anzeiger von anthropogener Aktivitäten zusammengefasst.

Clusteranalyse, zusammenfassende Übersichttabellen der Untersuchungsergebnisse vor Stau und nach Vollstau und auch Starplotdarstellungen geben einen Gesamtüberblick über Veränderungen im Untersuchungsgebiet wieder. Basierend auf all diesen Erkenntnissen konnten lokale und räumliche Zusammenhänge aufgezeigt werden.

\section{GESAMTBEURTEILUNG „2. UND 20. BEZIRK"}

Durch laufende Kontrolle der Wasserqualität der Donau und des Uferfiltrates wird das Eindringen von Kontaminationen bzw. Schadstoffen in den Aquifer verhindert. Sieben OnlineWasserqualitätsmessstationen von Donau und Donauuferfiltrat dienen als Vorwarnsystem. Neben chemischphysikalischen Parametern werden
Kohlenwasserstoffe, Ammonium und Organischer Kohlenstoff $1 / 4$-stündlich gemessen. Nicht näher definierbare schädliche Wasserinhaltsstoffe stellen ein großes Gefahrenpotenzial dar (MUSOLFF et al., 2007). Da es nicht möglich ist, jede einzelne Substanz $\mathrm{zu}$ analysieren, wird mittels Biomonitoring (Daphnien- bzw. MuschelMonitoring) deren Gefahrenpotenzial kontinuierlich erfasst. Bei Überschreitungen vordefinierter Grenzwerte der untersuchten Parameter schaltet die Grundwasserbewirtschaftung automatisch ab. Somit gelangt gegenüber dem "IST-Zustand“ vor Stauerrichtung nur noch chemisch „einwandfreies Wasser" zur Versickerung. Somit sind die bestimmenden Einflussfaktoren für den 2 . und 20 . Bezirk einerseits die Grundwasserbewirtschaftung und andererseits die Wasserspiegellagen des Donaukanals. Wie die Signifikanzuntersuchungen zeigen, ist der Nahbereich der Donau sowie der Bereich südlich der Reichsbrücke deutlich durch die Grundwasserbewirtschaftung beeinflusst, bei welcher ionenärmeres Uferfiltrat im Grundwasserkörper des 2. und 20. Bezirkes zur Versickerung gebracht wird. Dadurch zeigen leitfähigkeitsrelevante Parameter signifikante Verringerungen, wohingegen der Bereich nahe dem Donaukanal zu signifikant erhöhten mittleren Konzentrationen tendiert. Vor allem die Parameter $\mathrm{Na}$ trium, Kalium, Bor und Nitrit folgen diesem Trend. Des Weiteren wurde die Grundwasserqualität im Bereich des Wiener Praters (von der Donau bis zum Donaukanal) deutlich verändert. Auffallend ist, dass der Parameter Oxidierbarkeit im gesamten Gebiet eine signifikante Erhöhung aufweist, wobei die Sauerstoffkonzentration bzw. Sättigung ebenfalls signifikant erhöht ist. Aufgrund der veränderten Wasserqualitätsdaten lassen sich im 2. und 20. Bezirk die ursprünglichen Altarmbereiche der Donau, welche vor Donauregulierung als Flussbett oder Seitenarm dienten, erkennen. Grund hierfür ist die bessere Wegigkeit im Untergrund mit höheren Durchströmungsgeschwindigkeiten, wodurch entlang dieser Altarme typisch Donau-beeinflusstes Grundwasser bis weit in das Hinterland des 2. und 20. Bezirkes vordringen kann. 
ORIGINALARBEIT | Auswirkungen der Grundwasserbewirtschaftung auf die Grund- und Oberflächenwasserqualität

\section{LITERATUR}

DREHER, J.E., GUNATILAKA, A. (2001): Management of urban groundwater - Part I. Quantitative aspects. In: GRIEBLER, C., DANIELOPOL, D.L., GIBERT, J., NACHTNEBEL, H.P., NOTENBOOM, J. (Eds.) (2001): Groundwater Ecology - A Tool for Management of Water Resources, pp 196-212. European Commission Environment and Climate Programme, Energy, Environment and Sustainable Development. EUR 19887, Office for official publications of European Communities, Luxembourg.

GUNATILAKA, A., DREHER, J.E. (2001): Management of urban groundwater - Part II. Qualitative aspects. In: GRIEBLER, C., DANIELOPOL, D.L., GIBERT, J., NACHTNEBEL, H.P., NOTENBOOM, J. (Eds.) (2001): Groundwater Ecology - A Tool for Management of Water Resources, pp 213-229. European Commission Environment and Climate Programme, Energy, Environment and Sustainable Development. EUR 19887, Office for official publications of European Communities Luxembourg.
MUSOLFF, A. et al. (2007): Xenobiotics in groundwater and surface water of the city of Leipzig. Grundwasser Volume 12, Number 3.

SCHUSTER S. (1995-2006): Donaukraftwerk Freudenau, Jahresberichte 1995 bis 2006.

SCHUSTER, S., PICHER, W. (2006): Does the fully automated groundwater management system lastingly influence the water quality of the 2nd and 2oth district? 36th IAD Conference, Klosterneuburg and Vienna, 15 September 2006.

Anschrift des Verfassers: Dr. Stefan SCHUSTER, Technisches Büro - Ingenieurbüro für Chemie und Biologie, Franzensgasse 19/5, A-1050 Wien, E-Mail: tb-schuster@chello.at 